\title{
Media Exposure of Covid-19 and Mental Health
}

\author{
Yunqian Zhang ${ }^{1, \dagger}$, Yichen $\mathrm{Lyu}^{2, \dagger}$, Ruize $\operatorname{Sun}^{3, *, \dagger}$ \\ ${ }^{1}$ Department of Psychology, University of California Santa Cruz, 95060, United States \\ ${ }^{2}$ Department of Psychology, Pennsylvania State University, 16802, United States \\ ${ }^{3}$ Department of Psychology, University of California Santa Cruz, 95060, United States \\ *Corresponding author. Email: rsun18@ucsc.edu \\ These authors contributed equally.
}

\begin{abstract}
COVID-19's aggressive appearance has caught the whole world by surprise. With the development of science and technology, people can be easily exposed to the global pandemic situation through media exposure. However, the issue that whether excessive media exposure of Covid-19 or similar negative events will influence people's mental health and aggravate the severity of mental illness has not received sufficient researcher attention. In this study, we collected 8 articles related to the media exposure of Covid-19 and mental health by searching for the keywords such as "media exposure," "Covid-19/coronavirus," "mental illness", of which 6 studies conducted cross-sectional design with surveys and questionnaires, and 2 studies were meta-analysis. This review found that during the pandemic of Covid-19 in 2019, with the exposure of negative content, people's depression and anxiety increased, and the exposure time of negative social media information was also related to the degree of depression and anxiety. The conclusions drawn from the review paper can be used to identify potential factors that may change the psychological state of the public in the context of an epidemic and control the use of social media or filter the news. In addition, it can also provide information to psychotherapists and journalists on how to stabilize people's mental health and selectively broadcast the news in the event of a serious disaster.
\end{abstract}

Keywords: media exposure, Covid-19, coronavirus, mental health

\section{INTRODUCTION}

A novel Covid-19 outbreak broke out around the world at the end of 2019. Wuhan, China, has become the most severe disaster area since the pandemic's beginning [1]. Since then, all kinds of social media and news platforms have begun to report news about Covid-19 massively. People paid very close attention to those news reports because of fear and worry, and even panic. Hoarding large quantities of goods and food sources is also a way people coping their negative experiences. For example, they also went to the supermarket and drugstore to buy masks, alcohol and even toilet paper [2]. Although these reports seem to help people learn more about the pandemic, excessive news about Covid-19 might harm people's mental health.

China is the first place where the outbreak of Covid19 occurred. The government has introduced a plan of home quarantine. Due to the quarantine, people get more opportunities to be exposed to the pandemic through online channels. Three weeks after the outbreak, indoor facilities such as cinemas and shopping centers were closed, public transport was suspended, and the community was closely monitored [3]. The whole country was under blockade, and more than 50 million confirmed and suspected patients were isolated. In this situation, people may experience fear, loneliness, anxiety, and depression due to the social isolation, fear of infection and the negative information brought by social media they were exposed to every day [3]. Media exposure can be defined as "the extent to which audience members have encountered specific messages or classes of messages/media content" [4]. With the giant progress of modern science and technology, people's media exposure rate is gradually increasing, which means that social media exposure on people is being intensified. Pfefferbaum et al.'s study (2003) showed that after the explosion of Oklahoma City in 1995, the indirect interpersonal contact between radio and print media and emotional reaction of media reports could lead to posttraumatic stress reaction of children even far away from the explosion [5]. Furthermore, the exposure of flat media was more harmful than broadcasting. Considering 
that the negative effect of the media exposure of negative incidents can cause mental illness such as traumatic sequelae, we would like to investigate the association between the media exposure of COVID-19 and mental illness.

\section{METHOD}

For our research purpose, we conducted the following actions : 1. We used ProQuest as our database to collect the articles we reviewed. 2. We searched the keywords "Media exposure", "Covid-19," and "Mental illness" as the topic of the study. 3. When we collected specific articles and researched through keywords, we only considered articles about online media exposure. 4 . The article should only be written in English, and the year span of our selected articles is 2003-2021. This article reviews 8 articles about the relationship between media exposure of Covid-19 or analogous negative news exposure and mental health.

\section{QUESTIONNAIRE APPROACH}

Based on the 8 scholarly articles we found, 6 were cross-sectional studies, and the other two were metaanalyses. In the 6 cross-sectional studies, some scales and questionnaires were used to measure the level of anxiety, depression, or negative emotions, which were the World Health Organization Well-Being Index (WHO-5), Generalized Anxiety Disorder scale (GAD-7), 4-item Patient Health Questionnaire (PHQ-4), Self-Rating Anxiety Scale (SAS-20), 5-point Likert-type scale, 21item Depression Anxiety Stress Scale (DASS- 21) and The Positive and Negative Affect Scale (PANAS). As for the degree of exposure to social media, researchers had different ways to measure it. For instance, in the study by Riehm et al., participants were asked to report (1) the average time spent on social media (hours) and (2) the number of traditional media sources (radio, television, and newspapers) consulted to learn about the COVID-19 with self-reported mental distress. However, in another study by Gao et al. [6], participants were asked to indicate how often they had access to COVID-19 news and information on social media (such as Sina Weibo, Zhihu, Douban, WeChat, etc.) in the past one week, and the response options were: "never", "occasionally", "sometimes" "often" and "very often". The research methods are that the participants fill in the scales and questionnaires; meanwhile, the researchers ask questions or combine the two and then analyze the results. Because the psychological problems measured in each study were different, the scales used were also diverse. However, when screening the literature, we found that DASS-21 and Self Rating Depression Scale (SDS) were used more in measuring depression, and GAD-7 was the most used scale in measuring anxiety.

\section{THE NEGATIVE IMPACT OF SOCIAL MEDIA EXPOSURE ON MENTAL HEALTH}

Results in the eight academic literatures mostly illustrated that social media could have a negative impact on mental health. According to eight literatures, the negative effects were mainly focused on levels of depression and anxiety, two of the more obvious and easily measured mental health problems. We divided the results into four basic categories: 1) depression, 2) anxiety, 3) resilience, and the 4) role of social media in disseminating health information, methods of response to outbreaks and misinformation for comparison and analysis. The first is the level of depression. Three research on depressive symptoms were included in the review paper [7] [8] [9]. They examined the relationship between news exposure and the number of social media uses with the level of depression, respectively. The results showed that people's depression increased with increasing social media exposure [8]. The second aspect is the level of anxiety, and three papers were used for comparison [7] [10] [11]. People's anxiety levels rise when exposed to news about events that can cause public emergencies such as earthquakes and epidemics; However, there is no direct correlation between social media addictive behavior and anxiety levels unless people have moderate and severe levels of anxiety in the first place [6]. And symptoms of anxiety vary across age groups, with findings showing that younger people are more anxious than older people when receiving the same news about COVID-19 [11]. Resilience as a mental health factor represents people's psychological ability to withstand and recover from different situations, and the results showed that social media use could affect individuals' emotions and resilience; negative news decreases people's positive emotion and resilience, while positive news can increase people's positive emotion and resilience [12]. Finally, Tao et al.'s (2021) scoping view examined the importance of social media during the epidemic, and the results showed that social media data could help identify mental health problems at the population level. Due to the early outbreak of New Coronavirus disease and the use of social media in China (such as Sina micro-blog and WeChat), the results indicated that mental health problems in the Chinese population increased. Similar trends in mental health may also occur in other regions. This outcome aims to strengthen the reliability of the results of the previous seven papers.

\section{CORRELATION RESULTS}

Because the scales used in the eight studies were different, we compared the correlations of data analysis. Firstly, in the study on depression, Olagoke et al. (2020) also focused on the relationship between the media exposure of Covid-19 and mental illness and conducted a 
study on the relationship between COVID-19 news exposure and depression. The results showed that news exposure was positively associated with depressive symptoms. Zhong et al. (2021) conducted a study to determine the potential relationship between social media use (SMU) and mental health, including stress and secondary trauma. The evidence showed a positive association between Wuhan residents' depression level on SMU and the support they gained; however, the healthy behavior change cannot be predicted by informational support [9]. The study made by Riehm et al. (2020) mainly focused on whether exposure to social and traditional media during the rise of the COVID-19 pandemic was associated with mental distress among U.S. adults; in their study, 6329 participants who completed the online survey were randomly chosen from Understanding America Study; and the results showed that the positive association between the time spent on social media and the number of traditional media sources (television, radio, and newspapers) enhanced to understand coronavirus were related to the degree of mental pain. From the three experiments above that focused on depression levels, there was a positive relationship between people's depression levels and social media, i.e., depression levels increased with frequent use of social media.

For the anxiety aspects, the review paper has three scholarly articles. In Gao's experiment (2020), the WHO5 and GAD-7 were used, and the participants answered a questionnaire, and the results showed that social media exposure to coronavirus disease increased public anxiety. In another study by Liu et al. (2020), they focused on the causes of the anxiety level of Chinese people in the epidemic, especially under the exposure of the media to different COVID-19 information. The results showed that social media exposure to coronavirus disease increased public anxiety. Based on the online survey of Brailovskaia and Margraf (2021), there was a positive association between participants' burdens and social media use (SMU). The sense of control was negatively associated with burden and addictive SMU and became the mediator between those two variables; the association between burden and SMU was significant only in moderate and high levels of anxiety symptoms.

Regarding this part of resilience, Giri and Manurya's research (2021) focused on the features of social media news and psychological resilience (2021). The results showed that there were correlates with people's resilience and news title and the features of title are the moderators to those two variables, the resilience level was higher for the participants exposed to positive Covid-19 news titles, and the resilience will be affected by the exposure of negative Covid-19 news.

Lastly, According to Tsao et al. (2021), the study investigated the public attitude, identifying information epidemiology, assessing mental health, finding or predicting COVID-19 cases, analyzing the government's response to pandemic measures, and evaluating the quality of health information in preventive education videos are five aspects of the study [13]. This study showed the positive correlation between social media promotion of information about COVID-19 and public awareness of mental illness.

\section{LIMITATIONS OF PREVIOUS RESEARCH}

Since research on this kind of problem is few, we have not found much qualified literature when searching for it, and COVID-19 is a disease that has erupted in the last two years, some psychological problems may still be gradually forming, so the results of the studies may not be convincing and universal. Besides, due to home isolation during the epidemic period, many people may mistakenly regard the emptiness and boredom brought by the sudden isolation life as a psychological problem. In most studies, participants were asked to fill in the scales and questionnaires subjectively, so the results may not be accurate. On the other hand, whether conscious or unconscious, the media reports on COVID-19 will have their subjective color. Therefore, the information received by people may not be objective, which will also have various effects on people's mental health, then it will become a factor disturbing the results of studies.

Most of the limitations mainly come from the single research method. Most research methods are that the subjects fill in the scale subjectively, and there is no interview or more objective investigations. In addition, some people do not participate in the research or are unwilling to reveal the real situation for various reasons. The experimental results become more diverse and unreliable.

Other limitations include that those researchers only sampled people in their own countries. The experience of participants was also limited since the studies were conducted early. It is not easy to judge the causal relationship between social media exposure and mental health since they were almost all cross-sectional studies.

\section{DISCUSSION}

According to the above review, during the COVID-19 epidemic, the number of depression and anxiety in negative social media information exposure increased. In addition, the time of exposure to negative social media information was also related to the degree of depression and anxiety.

In modern society, the impact of this global plague on people is very serious. Similarly, there are many difficulties in epidemic prevention and control. Due to the development of science and technology, social media occupies a certain position in people's lives. Through 
social media exposure, people are exposed to much information every day. The information brought to people by social media exposure in the epidemic is not satisfactory. Suggestions and announcements from various public institutions such as health organizations and governments on maintaining personal safety and avoiding the spread of infection are mainly disseminated through the media. Therefore, although media exposure will lead to increased psychological problems, it is not feasible to leave the media. However, it is hopeful that a study shows that in the early stage of the epidemic, social media brings more negative emotions, but over time, this negative emotion will gradually improve and even appear positive emotions [1]. According to the existing research results, social media exposure will more or less affect people's mental health, which may cause anxiety, depression or negative emotions. However, over time and the epidemic, social media exposure can lead to positive emotions.

\section{LIMITATIONS AND FUTURE DIRECTION}

Because Covid-19 is a relatively novel virus, there is very limited literature on it, especially the focus of this review paper is on people's mental health. Therefore, the relevant literature found was very limited and repetitive. The review paper cited literature mainly from 2019 to 2021, a very short period that lacked longitudinal studies to give the review paper more resources to compare people's mental health in the post-epidemic era and related social media reports. Similarly, based on the scarcity of existing studies, the sources of this review paper were mostly from university library resources; the related studies may be limited to the level of college students; more advanced or sophisticated studies were not included in the review paper, so there is a limitation in data collection. In addition, the paper also has a study scope limitation. As mentioned before, mental health and media exposure are very broad variables, and this review paper does not limit the scope to a specific social media or a mental health issue. For example, in Zhong's study (2021), the social media that emerged was China's WeChat, a social media platform that is more for chatting purposes than for spreading news; Giri and Manurya's experiment pinpointed social media to news headlines; Tsao et al.'s study (2021) included several social media platforms such as CNN, New York Times and Washington Post, etc. In the case of a particularly broad scope, there are more potential research compounds such as different regions, countries, or the complexity of information reception.

\section{CONCLUSION}

From the results, social media exposure has a great influence in the epidemic, which can affect people's mental health. Therefore, the significance of this article is to make people aware of the "complications" of COVID19 , and also achieve many purposes through social media exposure in the epidemic, such as reminding people of the areas with serious epidemic and unified control.

Social media exposure has become the main way for the public to obtain information about the pandemic, and it plays a very important role. Through our research, we have found that people become more dependent on their phones and then develop addictive behaviors when they encounter catastrophic events like Covid-19. In addition to the most basic behavioral issues, the content attributes of social media messages can also affect people's mental health; in our study, reported negative news such as the number of deaths from the epidemic led to increased anxiety and depression; meanwhile, people's reliance on cell phones was also more severe. Moreover, the amount of information exposed on social media may make it difficult for people to distinguish between true and false information and find the information they need. In addition to disseminating public health information by the government's official media, some competent companies should also regulate misinformation and classify all information so that the public can find and browse it. This review aims to draw attention to social media addiction and the need to properly differentiate and filter the news content viewed in the event of an epidemic. When fluctuations in psychological conditions are detected, cell phone use and exposure to social media should be controlled in a timely manner.

\section{REFERENCES}

[1] Nie, X., Wang, Q., Wang, M., Zhao, S., Liu, L., Zhu, Y., \& Chen, H. (2020). Anxiety and depression and its correlates in patients with coronavirus disease 2019 in wuhan. International Journal of Psychiatry in Clinical Practice, doi:http://dx.doi.org.oca.ucsc.edu/10.1080/136515 01.2020.1791345.

[2] Garfin, D. R., Silver, R. C., \& Holman, E. A. (2020). The novel coronavirus (COVID-2019) outbreak: Amplification of public health consequences by media exposure. Health Psychology, 39(5), 355357. http://dx.doi.org/10.1037/hea0000875.

[3] Ren, X., Huang, W. L., Pan, H. P., Huang, T. T., Wang, X. W., Ma, Y. C. (2020). Mental Health During the Covid-19 Outbreak in China: a MetaAnalysis. Psychiatr $Q$ 91, 1033-1045. https://doi.org/10.1007/s11126-020-09796-5.

[4] Slater, M. D. (2004). Operationalizing and Analyzing Exposure: The Foundation of Media Effects Research. Journalism \& Mass Communication Quarterly, 81(1), 168-183. https://doi.org/10.1177/107769900408100112. 
[5] Pfefferbaum, B., Seale, T. W., Brandt, E. N., Jr., Pfefferbaum, R. L., Doughty, D. E., \& Rainwater, S. M. (2003). Media exposure in children one hundred miles from a terrorist bombing. Annals of Clinical Psychiatry, 15(1), 1-8. http://dx.doi.org.oca.ucsc.edu/10.1023/A:10232938 24492.

[6] Gao J, Zheng P, Jia Y, Chen H, Mao Y, Chen S, et al. (2020) Mental health problems and social media exposure during COVID-19 outbreak. PloS ONE 15(4): e0231924. https://doi.org/ 10.1371/journal.pone.0231924.

[7] Olagoke, A. A., Olagoke, O. O., \& Hughes, A. M. (2020). Exposure to coronavirus news on mainstream media: The role of risk perceptions and depression. British Journal of Health; Psychology, 10.

doi:http://dx.doi.org.oca.ucsc.edu/10.1111/bjhp.12 427.

[8] Riehm, K. E., Holingue, C., Kalb, L. G., Bennett, D., Kapteyn, A., Jiang, Q., Veldhuis, C. B., Johnson, R. M., Fallin, M. D., Kreuter, F., Stuart, E. A., \& Thrul, J. (2020). Associations Between Media Exposure and Mental Distress Among U.S. Adults at the Beginning of the COVID-19 Pandemic. American Journal of Preventive Medicine, 59(5), 630-638.

https://doi.org/10.1016/j.amepre.2020.06.008.

[9] Zhong, B., Huang, Y., \& Liu, Q. (2021). Mental health toll from THE CORONAVIRUS: Social media usage reveals Wuhan residents' depression and secondary trauma in the COVID-19 outbreak. Computers in Human Behavior, 114, 106524. https://doi.org/10.1016/j.chb.2020.106524.

[10] Brailovskaia, J., \& Margraf, J. (2021). The relationship between burden caused by CORONAVIRUS (Covid-19), addictive social media USE, sense of control and anxiety. Computers in Human Behavior, 119, 106720. https://doi.org/10.1016/j.chb.2021.106720.

[11] Liu, M., Zhang, H., \& Huang, H. (2020). Media exposure to COVID-19 information, risk perception, social and geographical proximity, and self-rated anxiety in China. BMC Public Health, 20(1), 1649-1649. https://doi.org/10.1186/s12889020-09761-8.

[12] Giri, S. P., \& Maurya, A. K. (2021). A neglected reality of mass media during covid-19: Effect of pandemic news on individual's positive and negative emotion and psychological resilience. Personality and Individual Differences, 180, 110962. https://doi.org/10.1016/j.paid.2021.110962.
[13] Tsao, S. F., Chen, H., Tisseverasinghe, T., Yang, Y. Li, L. H., Butt. Z. A. (2021). What social Media told us in the time of COVID-19: a scoping review. The Lancet Digital Health. https://doi.org/10.1016/S2589-7500(20)30315-0. 